\section{Reply to: Node Micrometastases Detection After Neoadjuvant Chemotherapy in Breast Cancer}

\section{TO THE EDITORS:}

We appreciate the comments by Dimosthenis Ziogas and colleagues concerning our report, recently published in the Annals of Surgical Oncology.

In recent multigene assays, the prognosis and treatment effect in breast cancer patients were predicted, not by clinicopathologic factors, but by multiple gene expression associated with these factors. These approaches contributed a great deal to the improvement of prognostic prediction. In the near future, multigene assays for breast cancer patients will identify low-risk patients not necessarily needing systemic treatment. Furthermore, more personalized therapies will be required in high-risk patients diagnosed as requiring systemic treatment. Therefore, in next-generation systemic treatment for primary breast cancer patients, prediction of treatment effect and confirmation of sufficiency or deficiency of treatment will be essential. Choice of optimal therapy using multigene assays will be crucial for these treatments. Furthermore, if patients are diagnosed as deficient in the confirmation of treatment effect, secondary systemic treatment will be needed. A concept of neoadjuvant treatment, the confirmation of treatment efficacy using target lesions in vivo, should be reevaluated in the near future. Thus, the choice of optimal therapy using multigene assays, the confirmation of treatment effect, and the correction of treatment will be essential in next-generation breast cancer treatment.

Recent study using multigene assays reported that invasion ability was strongly associated with survival in estrogen receptor (ER)-negative breast cancer patients. In neoadjuvant chemotherapy, pathologically complete remission of cytologically proven axillary lymph nodes metastases (ALN-pCR) was more correlated with survival than pathologically complete remission of main tumor. Therefore, axillary metastases may be the most relevant targets for confirmation of treatment efficacy in vivo in ERnegative breast cancer patients.

In our evaluation of axillary metastases using multislice sectioning, ALN-mic and ALN-mac were strongly associated with poor survival in ER-negative breast cancer patients. As discussed in our paper, these patients with ALN-mic and ALN-mac after neoadjuvant treatment may require secondary systemic treatment. Furthermore, patients with ALN-pCR and ALN-itc have good prognosis and may not need further treatment. We hope that our axillary evaluation will be applied for this confirmation and the correction of next-generation breast cancer treatment.

Masahiro Sakakibara, MD, Takeshi Nagashima, MD, and Masaru Miyazaki, MD

Department of General Surgery, Chiba University

Graduate School of Medicine, Chiba, Japan

e-mail: sakaki@faculty.chiba-u.jp

Published Online: 15 October 2009

(C) Society of Surgical Oncology 2009 